\title{
Diffuse idiopathic skeletal hyperostosis (DISH): relation to vertebral fractures and bone density
}

\author{
G. Diederichs $\cdot$ F. Engelken $\cdot$ L. M. Marshall • \\ K. Peters • D. M. Black • A. S. Issever • \\ E. Barrett-Connor • E. Orwoll • B. Hamm • T. M. Link • \\ Osteoporotic Fractures in Men (MrOS) Research Group
}

Received: 20 May 2010 /Accepted: 3 September 2010 / Published online: 30 September 2010

(C) The Author(s) 2010. This article is published with open access at Springerlink.com

\begin{abstract}
Summary Radiographs and spinal bone mineral density (BMD) were evaluated from 342 elderly men regarding possible effects of diffuse idiopathic skeletal hyperostosis (DISH) on vertebral fractures and densitometry measurements. Prevalent vertebral fractures were more frequent among men with DISH compared to men with no DISH even after fracture prevalence was adjusted for BMD. Paravertebral calcifications should be considered in patients with DISH when interpreting BMD measurements because both dual X-ray absorptiometry (DXA) and quantitative CT (QCT) densitometry may not be reliable.

Introduction The purpose of this study is to evaluate the prevalence of DISH in older men and its association with vertebral fractures and with BMD determined by DXA and QCT.

Methods Lateral radiographs of the spine were analyzed in a sample of 342 men aged $\geq 65$ years participating in the MrOS Study concerning the presence and grade of DISH and vertebral fractures. Lumbar BMD was measured by
\end{abstract}

\section{G. Diederichs $\cdot$ A. S. Issever $\cdot$ T. M. Link}

Department of Radiology and Biomedical Imaging,

Musculoskeletal and Quantitative Imaging Research Group,

University of California,

China Basin Landing, 185 Berry Street,

San Francisco, CA 94107, USA

G. Diederichs $(\bowtie) \cdot$ F. Engelken $\cdot$ A. S. Issever $\cdot$ B. Hamm Department of Radiology, Charité-Universitätsmedizin Berlin, Charité Campus Mitte, Chariteplatz 1,

10117 Berlin, Germany

e-mail: gerd.diederichs@charite.de

\section{M. Marshall}

Department of Orthopaedics and Rehabilitation and Bone and

Mineral Unit, Department of Medicine,

Oregon Health and Science University,

Portland, OR, USA both DXA (areal, grams per square centimeter) and QCT (volumetric, grams per cubic centimeter). The association between DISH, BMD, and presence of fractures was studied using $\chi^{2}$ and $t$ tests.

Results DISH was present in 52\% (178/342) of the men. Men with DISH were older (mean, 75.1 vs $73.3, p<0.05$ ) and more likely to have prevalent fractures ( $28 \%$ vs $20 \%, p<p=0.09$ ). BMD assessed with DXA (1.08 vs $\left.1.00 \mathrm{~g} / \mathrm{cm}^{2}, p \leq 0.0001\right)$, but not with QCT $(0.11$ vs $0.11 \mathrm{~g} / \mathrm{cm} 3, p=0.65)$, was significantly higher in men with DISH compared to men without DISH. Significantly lower BMD of men with both DISH and fractures compared to men with DISH but without fractures was only detected by QCT $(-25 \%$, 0.09 vs $0.12, p<0.05)$. Both DXA BMD and QCT BMD were significantly higher in severe lumbar DISH $(+22 \%$ and $+31 \%, p<0.0001$ ), respectively.

Conclusion DISH was associated with a higher prevalence of vertebral fractures in elderly men. Lumbar ossifications related to DISH should be considered when interpreting BMD measurements to predict their fracture risk.

K. Peters $\cdot$ D. M. Black

San Francisco Coordinating Center, University of California,

San Francisco and the California Pacific Medical Center Research Institute,

San Francisco, CA, USA

E. Barrett-Connor

Division of Epidemiology, Department of Family and Preventive

Medicine,

University of California,

9500 Gilman Drive, La Jolla,

San Diego, CA 92093-0607, USA

E. Orwoll

Bone and Mineral Unit,

Oregon Health and Science University,

3181 SW Sam Jackson Park Road,

Portland, OR 97239, USA 
Keywords DISH · DXA · MrOS · QCT · Vertebral fracture

\section{Introduction}

Diffuse idiopathic skeletal hyperostosis (DISH) is a skeletal disorder of unknown etiology described in the elderly and characterized by abundant bone formation, ossification, and calcification of connective tissue in spinal and extraspinal sites. The vertebral findings were first described as "senile vertebral ankylosing hyperostosis" by Forestier and RotesQuerol [1]. The concept of DISH was later extended by Resnick et al. [2] to also include extraspinal manifestations. The prevalence of DISH ranges from $4 \%$ to $35 \%$ and varies across different study populations investigated and the diagnostic criteria used [3-5].

Spinal manifestations of DISH consist of craniocaudally oriented paravertebral and paradiscal bone formation and osteophytes with a predilection for the anterior longitudinal ligament. Spinal ossifications can be extensive and may lead to esophageal stenosis or neurological disorders. Controversy exists about the implications of vertebral ossifications for the mechanical stability of the spine. It has been suggested that the fused segments are more prone to fracture even after minimal trauma [6]. On the other hand, different studies have shown consistently higher bone mineral density (BMD) in patients with hyperostosis, implying a lower fracture risk [7-9]. All of these previous studies were performed with dual energy X-ray absorptiometry (DXA), which measures two-dimensional areal BMD as a sum of all attenuating tissues in the beam projection. Flowing ossifications may lead to overestimation of BMD values by DXA, limiting evaluation of fracture risk in these patients. It is not clear what BMD to expect in trabecular bone when measurement is performed using quantitative computed tomography (QCT), which allows separate measurement of trabecular bone and cortical bone of the spine in three dimensions, not influenced by surrounding osteophytes.

Knowledge of fragility fractures and BMD in association with DISH is limited. The goals of this study were to evaluate the prevalence of DISH in association with presence and absence of vertebral fractures and to analyze BMD determined by DXA and QCT in relation to vertebral DISH and fractures.

\section{Materials and method}

Study participants

A total of 342 participants were randomly selected from 5,995 men 65 years or older participating in the prospective multicenter, observational Osteoporotic Fractures in Men (MrOS) Study; details of MrOS have been published previously $[10,11]$. The baseline examinations were performed from March 2000 to April 2002 at six clinical centers in the United States: Birmingham, AL; Minneapolis, MN; Palo Alto, CA; Pittsburgh, PA; Portland, OR; and San Diego, CA. Briefly, the inclusion criteria included: (1) ability to walk, (2) absence of bilateral hip replacement, (3) ability to provide self-reported data, (4) residence near a clinical site for the duration of the study, (5) absence of a medical condition that would result in imminent death, and (6) ability to understand and sign an informed consent. The protocol and consent forms for MrOS were approved by the institutional review boards at each of the participating institutions. All participants provided written informed consent.

Imaging and image analysis

Lateral radiographs of the thoracic and lumbar spine were available in all 342 participants at baseline. Thoracic radiographs were centered at $\mathrm{T} 7$ and lumbar radiographs at $\mathrm{L} 3$. All radiographs were reviewed in consensus by two radiologists experienced in musculoskeletal radiology (TML, 20 years of experience; GD, 4 years of experience).

\section{Assessment of DISH}

Two scoring systems were used to diagnose spinal DISH from T4 to S1: (1) Resnick et al. [2] defined DISH as the presence of four or more vertebral bodies with continuous ossification of the anterior spinal ligaments and absence of degenerative disc disease. (2) Mata et al. [12] developed a scoring system to grade DISH from 0 to 3 based on ossifications at each disc space level, where 0 is defined as no ossification, $1=$ ossification without bridging, $2=$ ossification with incomplete bridging, and $3=$ complete bridging of the disc space. Additionally, a grade 4 was introduced for severe ossifications and extensive bridging of more than $1 \mathrm{~cm}$ thickness. Presence of DISH was defined according to Mata as a grade of 2,3, or 4 at three or more consecutive disc space levels.

To analyze the association of lumbar DISH-related ligamentous ossifications in the lumbar segments on DXA and QCT measurements, the men were separated into three subgroups by summarizing the total Mata scores from each lumbar segment L1 to L3: no relevant lumbar DISH=Mata score $0-3$, moderate lumbar DISH=Mata score 4-6, and severe lumbar DISH=Mata score $>7$.

Assessment of vertebral fractures

Fracture status of T4 to L5 was assessed semiquantitatively on the lateral radiographs as described by Genant et al. [13]. Vertebral fracture deformities were graded as $0=$ none, $1=$ mild $(20-25 \%$ reduction in vertebral height), $2=$ moderate 
(25-40\% reduction in vertebral height), and $3=$ severe ( $>40 \%$ reduction in vertebral height). Vertebral deformities grade 2 and grade 3 on the baseline radiographs were defined as prevalent vertebral fractures only when osteoporotic endplate depression with or without typical appearance of wedge or biconcave shape was present. Vertebral deformities that were judged most likely of lytic or posttraumatic origin were classified separately.

\section{Bone mineral density measurements}

As previously described, areal BMD measurements in grams per square centimeter of the L1-L4 were obtained using the same model fan beam dual-energy X-ray absorptiometry machine at all clinical sites (QDR 4,500 W, Hologic Inc., Bedford, MA) at baseline [14]. Quality assurance with review of the DXA scans was performed at the coordinating center on random subsets of scans and on problematic scans identified by technicians at the centers. Among the 342 lumbar DXA scans, measurements of a single vertebra were excluded in five participants due to poor image quality; the BMD values of the other three vertebrae were used to calculate mean lumbar BMD.

Trabecular BMD was analyzed using volumetric QCT scans according to methods previously described $[15,16]$. QCT scans were available from 192 subjects (56\%) because study resources at baseline supported QCT among two thirds $(3,785)$ of the cohort [17]. Briefly, hydroxyapatite equivalent densities in grams per cubic centimeter were obtained at baseline to measure BMD of the lumbar vertebrae L1 and L2. All scans were obtained using a standardized protocol and calibration standards. Scan range was from $5 \mathrm{~mm}$ above the L1 superior endplate to $5 \mathrm{~mm}$ below the L2 inferior endplate at scanner settings of $120 \mathrm{kVp}, 150 \mathrm{~mA}, 1-\mathrm{mm}$ slice thickness and $512 \times 512$ matrix in spiral reconstruction mode. All scans were transferred to the coordinating center for central quality review and image processing. The trabecular BMD of the central vertebral body was calculated by using semicircular 3D ROIs in the $10-\mathrm{mm}$ slice in the mid-vertebra section encompassing about $70 \%$ of the central vertebral body as proposed by Lang et al. [15]. If either the L1 or L2 values were set to a missing value, BMD was calculated at the other level.

\section{Other measurements}

At baseline, body weight and height were measured in participants wearing indoor clothing with shoes removed, using a standard protocol and regularly calibrated equipment. Weight and height were used to calculate the body mass index (BMI; kilogram per square meter).

A self-administered questionnaire was used to obtain information on demographic characteristics, lifestyle factors, and medical history. History of diabetes mellitus was obtained from self-report of diabetes diagnosed by a physician. Men were asked about their history of cigarette smoking, including ages at initiating and quitting and pack years of smoking was computed from their responses. Current alcohol consumption was reported and quantified in terms of usual drinks per day using an interviewer-administered questionnaire.

Also, severity of degenerative disc disease (DDD) was separately graded for the thoracic and lumbar spine from the radiographs as grade $0=$ none, $1=$ mild (minor osteophytes), $2=$ moderate (large osteophytes, significant disc space narrowing), and $3=$ severe (absence of disc space, significant sclerosis). The prevalence of Scheuermann's disease, scoliosis, and ankylosing spondylitis was assessed using the typical imaging features as previously described [16].

\section{Statistical analysis}

Descriptive statistics of the study group and prevalence of DISH and vertebral fractures were calculated. Distributions of baseline characteristics among participants with and without DISH were compared using $\chi^{2}$ tests for categorical variables and $t$ tests for continuous variables. BMD values derived from DXA and QCT measurements were compared within subgroups by $t$ tests and linear regression analysis. The influence of age and BMI on BMD was assessed with linear regression analysis and on fractures with logistic regression analysis. $\chi^{2}$ test was used to assess the association between fractures and lumbar DISH status. Agreement between the Mata and Resnick procedure was assessed with Kappa statistics. We used multivariable log-binomial regression models to estimate prevalence ratios (PR) and their 95\% confidence intervals ( $\mathrm{CI})$ as the measure of association between DISH and the prevalence of vertebral fractures [18, 19]. For each definition of DISH (Mata criteria, Resnick criteria) the PR are reported first with adjustment for age and DXA BMD and second with additional adjustment for BMI, diabetes history, and smoking pack years. For comparison, the PR were also estimated using QCT BMD among the 192 men with baseline QCT scans. PR greater than 1.0 indicated increased fracture prevalence among men with DISH compared to men without. The statistical analysis was performed with SPSS, version 17.0, for Mac (Chicago, IL) and SAS, version 9.2 for windows (Cary, NC).

\section{Results}

\section{Prevalence of DISH}

The mean age of these men was 74.2 years (range, 6591 years; SD, 6.1 years). The overall prevalence of DISH was $52 \%$ using the Mata criteria and $38 \%$ using the Resnick 
criteria (Table 1). Men with DISH were on average older and heavier than men without DISH. Diabetes history, smoking pack years, and current alcohol consumption varied little according to DISH status. Forty- nine of the 178 men (28\%) classified positive for DISH using the Mata criteria were negative for DISH according to the Resnick system $(\kappa=0.72, p<0.05)$. Among the men diagnosed with DISH using the Mata criteria, vertebral ligamentous calcifications were predominantly present at the thoracic spine with a peak between T8-T10 (Fig. 1a). The upper thoracic spine and the lower lumbar spine were less commonly affected.

Bone mineral density measurements and fracture prevalence

Overall, lumbar spine BMD measurements by DXA were nearly $10 \%$ higher in the DISH group defined by either the Mata or the Resnick criteria (Table 2). For example, by the Mata criteria, mean DXA BMD was $1.08 \pm 0.19 \mathrm{~g} / \mathrm{cm}^{2}$ among men with DISH compared to $1.00 \pm 0.16 \mathrm{~g} / \mathrm{cm}^{2}$ among men without DISH. In contrast, mean QCT values did not differ significantly according to DISH status. Vertebral fracture prevalence was 1.4 times greater among men with DISH (28\%) compared to men without DISH (20\%) using the Mata criteria; however, using the Resnick criteria, fracture prevalence did not differ materially according to DISH status. In the multivariable regression analyses, vertebral fracture prevalence remained about 1.5 times greater among men classified with DISH by the Mata criteria compared to men without DISH ( $\mathrm{PR}=1.5 ; 95 \% \mathrm{CI}, 1.0-2.2)$ when adjusted for DXA BMD. In the analyses restricted to men with QCT BMD, the magnitude of the PR was similar, but the $95 \%$ CI were wider presumably because of the decreased sample size. The PR indicate that variation in BMD, age or other factors did not account for the positive association between DISH and fracture prevalence. In contrast, DISH classified according to the Resnick criteria was not associated with vertebral fracture prevalence.

\section{Characteristics of vertebral fracture}

Vertebral fractures were primarily grade 2 and 3. Among men with vertebral fractures, $28 \%(23 / 83)$ had two vertebral fractures and $8 \%$ (seven out of 83 ) had more than three fractures. Other vertebral deformities not counted as fractures were uncommon; seven men $(2.1 \%)$ had posttraumatic deformities and three men $(0.9 \%)$ had deformities

Table 1 Characteristics of the study population

\begin{tabular}{|c|c|c|c|c|c|}
\hline \multirow[t]{2}{*}{ Variable } & \multirow[b]{2}{*}{ All } & \multicolumn{2}{|l|}{ Mata } & \multicolumn{2}{|l|}{ Resnick } \\
\hline & & DISH & Non-DISH & DISH & Non-DISH \\
\hline $\begin{array}{l}\text { Number of } \\
\text { cases }(\%)\end{array}$ & 342 & $178(52)$ & $164(48)$ & $129(38)$ & $213(62)$ \\
\hline $\begin{array}{l}\text { Age in years; mean } \pm \mathrm{SD} \\
\text { (range) }\end{array}$ & $74.2 \pm 6.1(65-91)$ & $75.1 \pm 6.1^{\mathrm{a}}(65-91)$ & $73.3 \pm 6.0(65-90)$ & $75.2 \pm 6.2^{\mathrm{a}}(65-90)$ & $73.6 \pm 6.1(65-91)$ \\
\hline $\begin{array}{l}\mathrm{BMI} \mathrm{kg} / \mathrm{m}^{2} ; \text { mean } \pm \mathrm{SD} \\
\text { (range) }\end{array}$ & $27.5 \pm 3.5(19.3-42.6)$ & $27.8 \pm 3.6(20.2-42.6)$ & $27.1 \pm 3.4(19.3-40.7)$ & $28.1 \pm 3.5^{\mathrm{a}}(20.7-42.6)$ & $27.1 \pm 3.4(19.3-40.7)$ \\
\hline Vertebral fractures $(\%)$ & $83(24)$ & $50(28)$ & $33(20)$ & $35(27)$ & $48(23)$ \\
\hline Diabetes (\%) & $46(13)$ & $25(14)$ & $21(13)$ & $19(15)$ & $27(13)$ \\
\hline Current smoker (\%) & $5(1)$ & $2(1)$ & $3(2)$ & $2(2)$ & $3(1)$ \\
\hline Past smoker (\%) & $191(56)$ & $109(61)$ & $82(50)$ & $81(63)$ & $110(52)$ \\
\hline Never smoked (\%) & $146(43)$ & $67(38)$ & $79(48)$ & $46(36)$ & $100(47)$ \\
\hline$>0$ to $<25$ Pack years & $107(31)$ & $58(33)$ & $49(30)$ & $44(34)$ & $63(30)$ \\
\hline$\geq 25$ to $<50$ Pack years & $48(14)$ & $29(16)$ & $19(12)$ & $22(17)$ & $26(12)$ \\
\hline$\geq 50$ Pack years & $40(12)$ & $23(13)$ & $17(10)$ & $17(13)$ & $23(11)$ \\
\hline Non-drinker & $112(33)$ & $58(33)$ & $54(33)$ & $41(32)$ & $71(33)$ \\
\hline$<7$ Drinks per week & $139(41)$ & $67(38)$ & $72(44)$ & $50(39)$ & $89(42)$ \\
\hline 7 to $<14$ Drinks per week & $43(13)$ & $24(13)$ & $19(12)$ & $17(14)$ & $26(12)$ \\
\hline$\geq 14$ Drinks per week & 48 (14) & $29(16)$ & $19(12)$ & $21(16)$ & $27(13)$ \\
\hline
\end{tabular}

Descriptive statistics of the MrOS subset of 342 randomly selected men age $\geq 65$ years. The diagnostic criteria of Mata [12] and Resnick [2] were used for classification of DISH from lateral radiographs

${ }^{\mathrm{a}} t$ test $(p<0.05)$ 


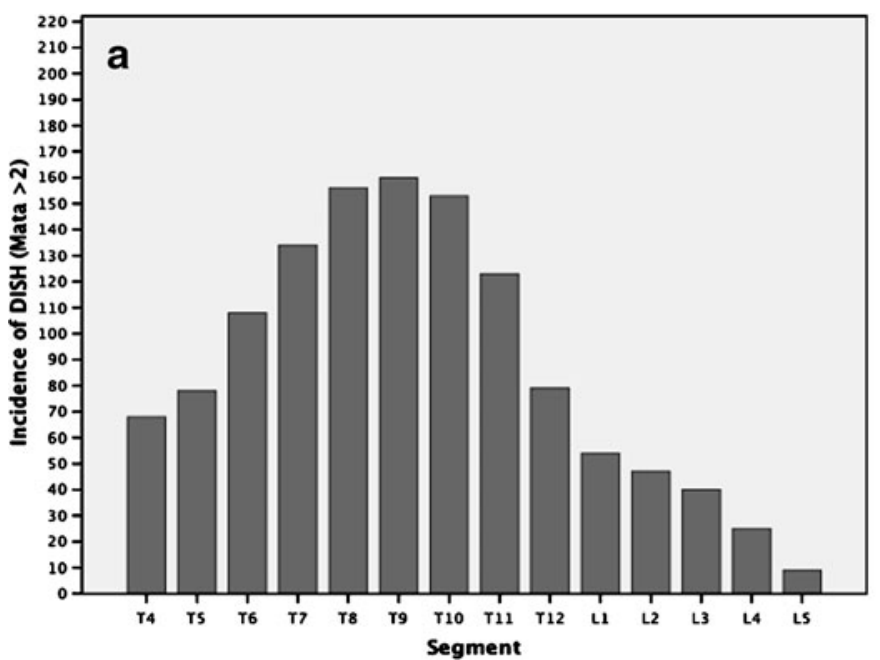

Fig. 1 Manifestations of DISH according to the Mata classification in the total study population (a) and prevalence of vertebral fractures (b) per spinal segment from T4 through L5. Ligamentous ossifications in

likely due to degenerative disease. Lytic lesions were found in two men $(0.6 \%)$.

In the 50 men with DISH who had fractures, $70 \%$ (35/ 50 ) were localized at either T12 or L1 while most other fractures occurred at the lumbar spine (Fig. 1). This distribution of spinal fracture sites was similar to that seen in men without DISH.

\section{b}

$25-1$

$23-$

$22-$

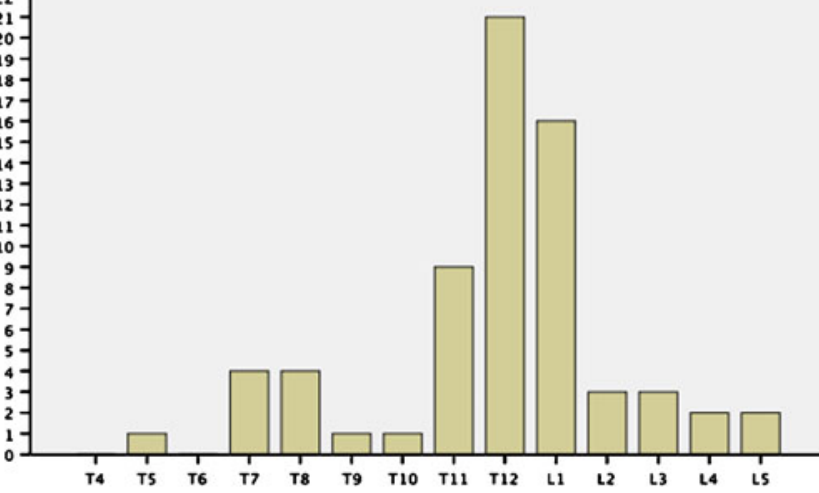

Segment

DISH mainly involved the middle and lower thoracic spine (a). More than half of all fractures affected T12 and L1 (b).

Interrelationships of DISH, bone mineral density measurements, and fractures

Lumbar spine DISH according to the Mata criteria were as follows: 123/178 (69\%) subjects showed no relevant signs of lumbar DISH, 34 (19\%) had moderate, and $21(12 \%)$ severe lumbar ossifications at the L1-3 levels (Table 3). To

Table 2 Densitometry in relation to DISH and fractures

\begin{tabular}{|c|c|c|c|c|c|c|}
\hline & \multicolumn{2}{|l|}{ Mata } & \multirow[t]{2}{*}{$P$ value } & \multicolumn{2}{|l|}{ Resnick } & \multirow[t]{2}{*}{$P$ value } \\
\hline & $\begin{array}{l}\text { DISH } \\
(n=178)\end{array}$ & $\begin{array}{l}\text { No DISH } \\
(n=164)\end{array}$ & & $\begin{array}{l}\text { DISH } \\
(n=129)\end{array}$ & $\begin{array}{l}\text { No DISH } \\
(n=213)\end{array}$ & \\
\hline \multicolumn{7}{|c|}{ DXA BMD $\left(\mathrm{g} / \mathrm{cm}^{2}\right)$} \\
\hline Mean \pm SD & $1.08 \pm 0.19$ & $1.00 \pm 0.16$ & $<0.0001$ & $1.10 \pm 0.19$ & $1.01 \pm 0.16$ & $<0.0001$ \\
\hline Range & $0.62-1.69$ & $0.60-1.57$ & & $0.62-1.69$ & $0.60-1.57$ & \\
\hline \multicolumn{7}{|c|}{ QCT BMD $\left(\mathrm{g} / \mathrm{cm}^{3}\right)^{\mathrm{a}}$} \\
\hline Mean $\pm \mathrm{SD}$ & $0.11 \pm 0.04$ & $0.11 \pm 0.03$ & 0.65 & $0.11 \pm 0.04$ & $0.11 \pm 0.03$ & 0.46 \\
\hline Range & $0.02-0.20$ & $0.04-0.22$ & & $0.04-0.20$ & $0.02-0.22$ & \\
\hline \multicolumn{7}{|l|}{ Vertebral fracture } \\
\hline Number (\%) & $50(28)$ & $33(20)$ & 0.09 & $35(27)$ & $48(23)$ & 0.34 \\
\hline PR $(95 \% \text { CI })^{\mathrm{b}}$ & $1.5(1.0-2.2)$ & 1.0 & 0.06 & $1.3(0.9-1.9)$ & 1.0 & 0.19 \\
\hline PR $(95 \% \mathrm{CI})^{\mathrm{c}}$ & $1.5(1.0-2.2)$ & 1.0 & 0.04 & $1.4(0.9-2.1)$ & 1.0 & 0.09 \\
\hline PR $(95 \% \text { CI })^{\mathrm{d}}$ & $1.5(0.9-2.4)$ & 1.0 & 0.11 & $1.2(0.7-1.9)$ & 1.0 & 0.50 \\
\hline PR $(95 \% \text { CI })^{\mathrm{e}}$ & $1.4(0.8-2.3)$ & 1.0 & 0.21 & $1.3(0.8-2.2)$ & 1.0 & 0.36 \\
\hline
\end{tabular}

Distributions of bone mineral density and fracture according to DISH status and association of DISH with vertebral fracture among men ages $\geq$ 65 years. The diagnostic criteria of Mata [12] and Resnick [2] were used for classification of DISH from lateral radiographs

a 192 men in the sample had QCT BMD measures

${ }^{\mathrm{b}}$ Adjusted for age and DXA BMD

${ }^{\mathrm{c}}$ Adjusted for age, DXA BMD, body mass index, history of diabetes, pack years of smoking, and current alcohol consumption.

${ }^{\mathrm{d}}$ Adjusted for age and QCT BMD. Analyses are based on 46 vertebral fracture cases

${ }^{\mathrm{e}}$ Adjusted for age, QCT BMD, body mass index, history of diabetes, pack years of smoking, and current alcohol consumption 
Table 3 Influence of lumbar DISH on DXA BMD and QCT BMD

\begin{tabular}{lll}
\hline DXA vs QCT & $\begin{array}{l}\text { DXA BMD } \\
\text { mean } \pm \text { SD } \\
\left(\mathrm{g} / \mathrm{cm}^{2}\right)\end{array}$ & $\begin{array}{l}\text { QCT BMD } \\
\text { mean } \pm \text { SD BMD } \\
\left(\mathrm{g} / \mathrm{cm}^{3}\right)\end{array}$ \\
\hline Lumbar DISH grade 0 $(n=123)$ & $1.03 \pm 0.16$ & $0.104 \pm 0.034$ \\
Lumbar DISH grade I $(n=34)$ & $1.14 \pm 0.17$ & $0.110 \pm 0.033$ \\
Lumbar DISH grade II $(n=21)$ & $1.25 \pm 0.21$ & $0.141 \pm 0.043$ \\
\hline
\end{tabular}

Results of lumbar densitometry in the DISH subgroup (total $n=178$ ) according to severity of lumbar hyperostosis (according to Mata score [12])

further explore the association of DISH and vertebral fracture, we used linear regression to quantify the relationship between lumbar DISH severity and densitometry (Table 3; Fig. 2). Men with moderate and severe lumbar DISH had an average DXA BMD score that was 0.12 and $0.23 \mathrm{~g} / \mathrm{cm}^{2}$ higher than those with no lumbar ossifications $(+12 \%$ and $+22 \%$, both, $p<0.0001)$, respectively (Fig. $2 \mathrm{a}$ ). When assessed by QCT, BMD values were also higher for each grade of severity, but only differences between severe vs no lumbar DISH were significant $\left(+0.033 \mathrm{~g} / \mathrm{cm}^{3},+31 \%, p<\right.$ 0.0001) (Fig. 2b). Within the DISH subgroups, fracture prevalence was not associated with the grade of lumbar DISH; $30 \%$ (37/123) of the men with DISH with no lumbar manifestation had vertebral fractures, $24 \%$ (eight out of 34) of those with moderate lumbar manifestation had fractures, and $24 \%$ (five out of 21 ) of those with severe lumbar manifestation had fractures.

Among men who had both DISH and fractures, mean QCT BMD values were 25\% lower than men with DISH, but no vertebral fractures when assessed by QCT $(0.09 \pm$ 0.03 vs $0.12 \pm 0.04, p<0.05$ ), and $5 \%$ lower BMD when assessed by DXA $(1.04 \pm 0.16$ vs $1.10 \pm 0.19, p=0.057)$ (Table 4). QCT measurements also showed $18 \%$ lower BMD values in DISH subjects with fractures compared to non-DISH subjects without vertebral fractures $(0.09 \pm 0.03$ vs $0.11 \pm 0.03, p=0.178$ ), whereas DXA results showed slightly higher BMD values in men with DISH and fracture compared to men without DISH and fractures $(1.04 \pm 0.16$ vs $1.01 \pm$ $0.16, p=0.061$ ). Logistic regression analysis revealed that increasing DXA BMD by one point was associated with a decrease in the odds of fracture by $0.8(p<0.05$.) similar to the 0.76 decrease in odds of fracture associated with increasing QCT BMD by ten points $(p<0.05)$.

Other spine conditions

Mild DDD was observed in the thoracic spine of 97 (29\%) men and in the lumbar spine of $70(21 \%)$ men, moderate thoracic DDD in $23(7 \%)$, and moderate lumbar DDD in 63 $(19 \%)$. Severe thoracic DDD was observed in two $(1 \%)$ men and severe lumbar DDD in $40(12 \%)$ men. Only 17 $(5 \%)$ had signs of Scheuermann's disease and one $(0.3 \%)$ of ankylosing spondylitis.

\section{Discussion}

Both DISH and vertebral fractures were common in this cross-sectional study of older community-dwelling men. Almost 50\% had DISH and almost 25\% had at least one vertebral fracture. Vertebral fractures were more common in men with DISH assessed with the Mata criteria. Although men with DISH were more likely to have vertebral fractures, BMD values measured by DXA were significantly

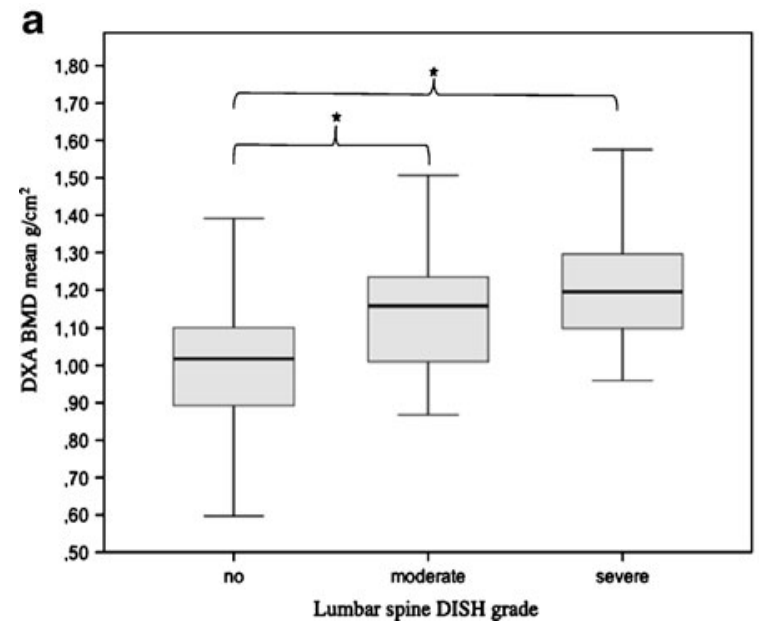

Fig. 2 Boxplots of BMD values obtained with DXA (a) and QCT (b) in relation to severity of lumbar DISH. Severity of lumbar manifestations of DISH-related paravertebral calcifications were graded using the Mata score for the segments L1-L3. Mata score 0-3 was graded as

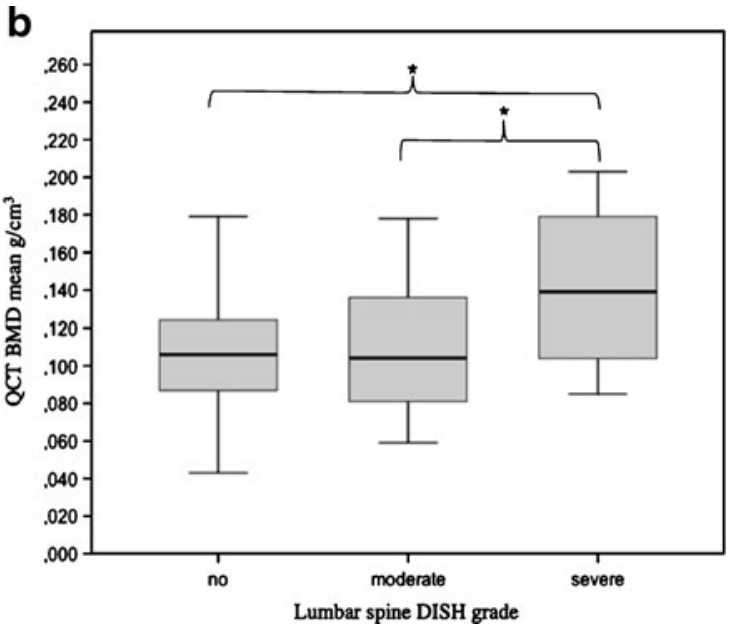

no lumbar DISH $(n=123)$, Mata score 4-6=moderate lumbar DISH $(n$ $=34)$, and Mata score $>7=$ severe lumbar DISH $(n=21)$. ${ }^{*}$ Significant differences 
Table 4 Densitometry in relation to DISH and fractures

Results of lumbar densitometry using QCT and DXA in DISH and non-DISH subgroups (Mata score [12]) in relation to vertebral fractures

\begin{tabular}{lccc}
\hline BMD QCT $\left(\mathrm{g} / \mathrm{cm}^{3}\right)$ & Fracture $(n=47)$ & No fracture $(n=145)$ & $P$ value \\
\hline DISH $(n=93)$ & $0.09 \pm 0.03$ & $0.12 \pm 0.04$ & 0.002 \\
No DISH $(n=99)$ & $0.11 \pm 0.03$ & $0.11 \pm 0.03$ & 0.691 \\
$P$ value & 0.178 & 0.105 & \\
BMD DXA $\left(\mathrm{g} / \mathrm{cm}^{2}\right)$ & Fracture $(n=83)$ & No fracture $(n=259)$ & $P$ value \\
DISH $(n=178)$ & $1.04 \pm 0.16$ & $1.10 \pm 0.19$ & 0.057 \\
No DISH $(n=164)$ & $0.95 \pm 0.16$ & $1.01 \pm 0.16$ & 0.061 \\
$P$ value & 0.021 & 0.0002 & \\
\hline
\end{tabular}

higher in DISH subjects compared to participants without DISH. Only QCT and not DXA showed lower BMD when comparing DISH subjects to those without DISH in groups with and without vertebral fractures. When assessing the association of densitometry with osteophytes at the site of measurement, both QCT and DXA values were significantly higher in subjects with severe lumbar ossifications. The positive association of DISH with vertebral fracture prevalence was independent of variation in BMD or other factors (Fig. 3).

The prevalence of DISH in our study is comparable to data reported in the literature, but prevalence estimates vary widely and vary with the classification system used and the population investigated [1, 3, 4, 20-23]. Kim et al. studied nearly 3,600 Korean men and women and found a low prevalence of DISH of only $2.9 \%$ using the Resnick criteria [4], possibly because the Korean men were about 10 years younger (mean age, 64 years) than the present cohort and of both genders. Other studies of younger men and women also found prevalences ranging between $2 \%$ and $4 \%[3,20$, 21]. The higher prevalence of DISH reported here is likely due to the subjects' older age and the fact that we only investigated men. For unknown reasons, DISH is up to seven times more common in men than women [4, 22]. Other studies, including only men report similar high prevalences of up to $30 \%[1,23,24]$.

It must be noted that the prevalence of DISH crucially depends on the classification criteria. In our study, the difference between the diagnosis of DISH according to the Mata or Resnick criteria may be partly explained by the fact that the Resnick criteria only classify segments with continuous ossifications as DISH while incomplete bridging between two vertebrae is sufficient to diagnose DISH according to the Mata criteria. This discrepancy affected 49 participants with only moderate manifestations of ligamentous ossifications, which were positive for DISH according to Mata while they were negative according to the Resnick criteria. To reduce the error in diagnosing and grading DISH, all radiographs were read by two experienced radiologists in consensus. It has been shown that interrater agreement is excellent when using both the Mata system (intraclass correlation coefficient $>0.83$ ) or the Resnick system $(\kappa=0.93)$ [12, 25].

This study attempts to determine how DISH is related to the prevalent vertebral fractures and to additionally quantify
Fig. 3 Lateral radiographs of a subject diagnosed with DISH according to the Mata [12] and Resnick [2] criteria. a Shows the spinal segments T7-T11 with bridging (arrows) and non-bridging (arrow head) osteophytes. The same subject had a vertebral fracture of T12 classified as a grade 3 fracture (star)
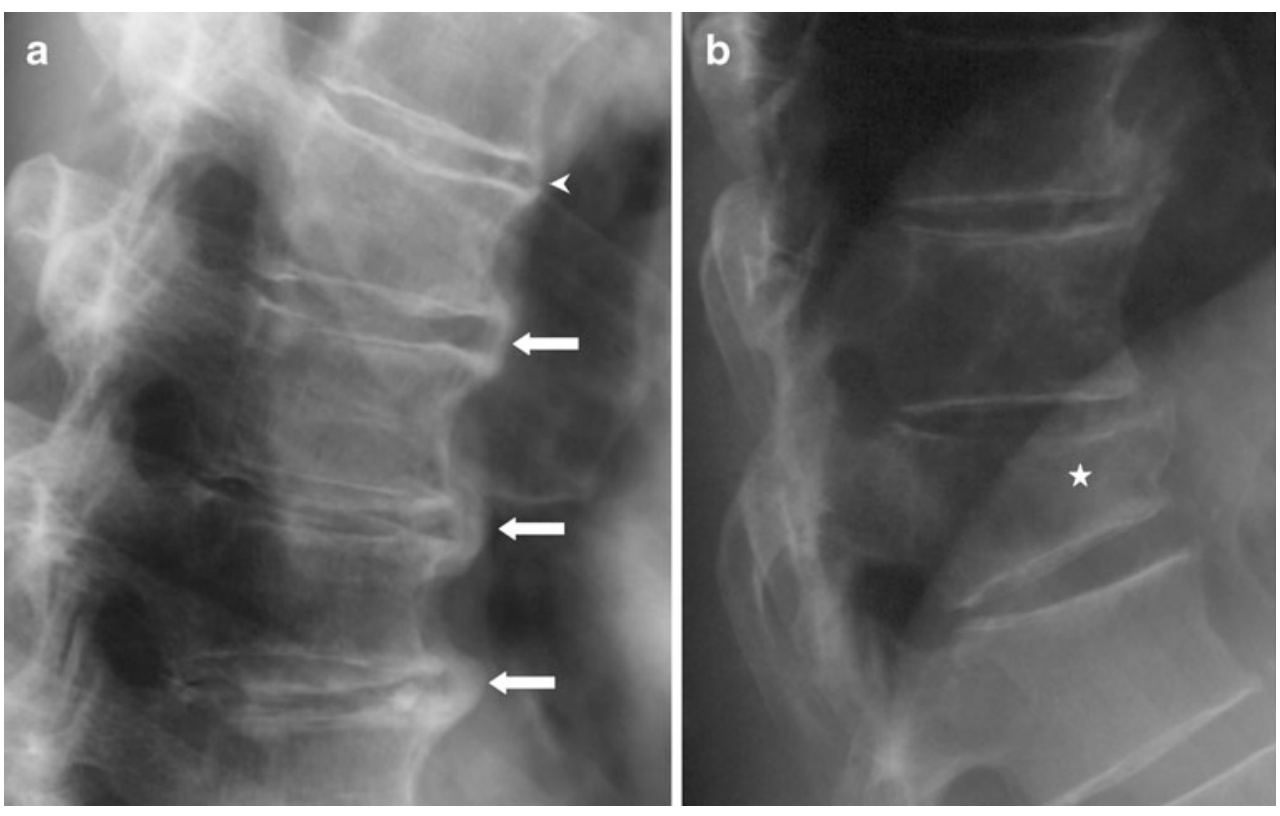
the impact of extraspinal ossification on BMD measurements. DXA and QCT BMD are widely used to assess fracture risk and make therapeutic decisions. Little is known about the accuracy of BMD measurement and their diagnostic implications in individuals with prevalent DISH, which may potentially affect these measurements. Resnick et al. described skeletal radiodensity in subjects with DISH appearing excessive in view of the patients' advanced age and that osteoporosis is not a feature of the disorder [23]; however, substantial controversy exists about the effects of spinal ligamentous calcifications in DISH on BMD results. Patients with ankylosing spondylitis showed significantly lower BMD measured by DXA at the lumbar spine and hip [26] while the opposite was found for patients with DISH $[7,8]$. The expected findings were previously illustrated in a case report of a man with severe lumbar DISH who had high DXA BMD values, which were interpreted as false negative because the same patient's distal radius BMD showed osteoporosis [9]. Higher DXA BMD values of the lumbar spine and hip were also reported in a study of 132 women with DISH [8]. In another study, individuals with spinal ligamentous ossifications also had higher BMD values of the peripheral skeleton [7]. The present study also shows that lumbar DISH is associated with higher BMD assessed DXA and QCT, suggesting that paraosseous calcifications in DISH may also affect trabecular BMD of the vertebrae in QCT measurements. On the other hand, overactive bone formation may occur in the trabecular bone as well.

In earlier studies, high BMD observed in patients with DISH was assumed to signify a lower fracture risk [8]; however, our findings suggest that men with DISH may have denser but more fragile bones leading to fractures. This result is difficult to comprehend as bone stability and fracture risk closely correlate with BMD. Therefore, other factors must explain reduced vertebral stability in subjects with DISH. Our results indicate that age, BMI, diabetes status, and smoking pack years do not explain the association of DISH and fracture prevalence. Thus, mechanical factors may provide a possible explanation. One possible explanation may be the ossification of the paraspinal ligaments, which reduces elasticity and impairs biomechanical competence, makes the spine more susceptible to biomechanical stress. Similar observations were made in ankylosing spondylitis, which is associated with a higher risk of vertebral fractures while the risk of peripheral fractures is not affected [27].

A possible clinical implication of our results is that patients with DISH of the lumbar spine should not undergo spinal BMD measurement with either QCT or DXA, as the findings appear to be of little value. As DISH primarily affects the spine, QCT measurement instead can be performed in the distal radius [28]; however, there is evidence that the increased fracture risk of the ankylosed spine is primarily attributable to changes in biomechanical properties $[27,29]$. This is why the prediction of the risk of fracture using BMD measurement in extraspinal body sites remains to be confirmed by further studies.

The pathogenesis of abnormal bone growth in DISH is not fully understood. It has been hypothesized that factors such as obesity, type 2 diabetes, drugs, and other metabolic disorders contribute to DISH pathogenesis [30-32]. An association of DISH with diabetes mellitus is not supported by our data. This was also reported from Sencan et al., who neither found significantly different prevalences of diabetes between DISH patients and controls [33]. BMI values in DISH and controls in a Hungarian study were similar to our data [21].

The present study has several strengths, including a large sample, multicenter community-based cohort, and standardized measurement of BMD by DXA and QCT and evaluation of DISH and vertebral fractures by specialized radiologists. Because this is a cross-sectional study, we cannot assume causality for the associations observed here. We did not use T-scores for a variety of reasons. First, $\mathrm{T}$-scores are dependent on a reference population, and they were not determined in the standard data set of the MrOS Study. Second, T-scores are not established for QCT measurements. Therefore, we used the actual BMD values as a reference for comparison of the densitometry techniques investigated. Also, this is a cross-sectional study, and the temporal relation between DISH and the occurence of vertebral fractures cannot be determined. Prospective studies are needed to determine wether DISH is a risk factor for subsequent vertebral fracture. Changes in biomechanical properties increase the risk of vertebral fractures but may also be associated with fractures of DISH-related osteophytes. Osteophyte fractures may also occur alone; however, a reliable diagnosis of fractured osteophytes requires an examination of the spine with $\mathrm{CT}$ or magnetic resonance imaging. We therefore did not analyze osteophyte fractures in the present study.

In conclusion, the results of this study demonstrate that (1) $52 \%$ of the elderly men in the study population had DISH, (2) vertebral fractures are more frequent among men with DISH, and (3) severe lumbar ossifications increase both QCT and DXA measurements. These results may have substantial implications for patient care because both DXA and QCT densitometry of the lumbar spine may not be reliable to assess fracture risk in the presence of DISH and because DISH may prove to be a new and previously unrecognized risk factor for fracture on older adults, particularly men.

Acknowledgements The Osteoporotic Fractures in Men (MrOS) Study is supported by National Institutes of Health funding. The following institutes provide support: the National Institute of Arthritis 
and Musculoskeletal and Skin Diseases (NIAMS), the National Institute on Aging (NIA), the National Center for Research Resources (NCRR), and NIH Roadmap for Medical Research under the following grant numbers: U01 AR45580, U01 AR45614, U01 AR45632, U01 AR45647, U01 AR45654, U01 AR45583, U01 AG18197, U01 AG027810, and UL1 RR024140. This manuscript has received the approval of the MrOS publications committee based on a review of its scientific content and data interpretation.

\section{Conflicts of interest None.}

Open Access This article is distributed under the terms of the Creative Commons Attribution Noncommercial License which permits any noncommercial use, distribution, and reproduction in any medium, provided the original author(s) and source are credited.

\section{References}

1. Forestier J, Rotes-Querol J (1950) Senile ankylosing hyperostosis of the spine. Ann Rheum Dis 9:321-330

2. Resnick D, Shaul SR, Robins JM (1975) Diffuse idiopathic skeletal hyperostosis (DISH): Forestier's disease with extraspinal manifestations. Radiology 115:513-524

3. Cassim B, Mody GM, Rubin DL (1990) The prevalence of diffuse idiopathic skeletal hyperostosis in African blacks. Br J Rheumatol 29:131-132

4. Kim SK, Choi BR, Kim CG et al (2004) The prevalence of diffuse idiopathic skeletal hyperostosis in Korea. J Rheumatol 31:2032-2035

5. Weinfeld RM, Olson PN, Maki DD, Griffiths HJ (1997) The prevalence of diffuse idiopathic skeletal hyperostosis (DISH) in two large American Midwest metropolitan hospital populations. Skeletal Radiol 26:222-225

6. Hendrix RW, Melany M, Miller F, Rogers LF (1994) Fracture of the spine in patients with ankylosis due to diffuse skeletal hyperostosis: clinical and imaging findings. AJR Am J Roentgenol 162:899-904

7. Di Franco M, Mauceri MT, Sili-Scavalli A, Iagnocco A, Ciocci A (2000) Study of peripheral bone mineral density in patients with diffuse idiopathic skeletal hyperostosis. Clin Rheumatol 19:188-192

8. Sahin G, Polat G, Bagis S, Milcan A, Erdogan C (2002) Study of axial bone mineral density in postmenopausal women with diffuse idiopathic skeletal hyperostosis related to type 2 diabetes mellitus. J Women's Health 11:801-804

9. Schwartz JB, Rackson M (2001) Diffuse idiopathic skeletal hyperostosis causes artificially elevated lumbar bone mineral density measured by dual X-ray absorptiometry. J Clin Densitom 4:385-388

10. Blank JB, Cawthon PM, Carrion-Petersen ML et al (2005) Overview of recruitment for the osteoporotic fractures in men study (MrOS). Contemp Clin Trials 26:557-568

11. Orwoll E, Blank JB, Barrett-Connor E et al (2005) Design and baseline characteristics of the osteoporotic fractures in men (MrOS) study - a large observational study of the determinants of fracture in older men. Contemp Clin Trials 26:569-585

12. Mata S, Chhem RK, Fortin PR, Joseph L, Esdaile JM (1998) Comprehensive radiographic evaluation of diffuse idiopathic skeletal hyperostosis: development and interrater reliability of a scoring system. Semin Arthritis Rheum 28:88-96

13. Genant HK, Wu CY, van Kuijk C, Nevitt MC (1993) Vertebral fracture assessment using a semiquantitative technique. J Bone Miner Res 8:1137-1148

14. Cauley JA, Fullman RL, Stone KL et al (2005) Factors associated with the lumbar spine and proximal femur bone mineral density in older men. Osteoporos Int 16:1525-1537
15. Lang TF, Li J, Harris ST, Genant HK (1999) Assessment of vertebral bone mineral density using volumetric quantitative CT. J Comput Assist Tomogr 23:130-137

16. Link TM, Guglielmi G, van Kuijk C, Adams JE (2005) Radiologic assessment of osteoporotic vertebral fractures: diagnostic and prognostic implications. Eur Radiol 15:1521-1532

17. Marshall LM, Lang TF, Lambert LC, Zmuda JM, Ensrud KE, Orwoll ES (2006) Dimensions and volumetric BMD of the proximal femur and their relation to age among older U.S. men. J Bone Miner Res 21:1197-1206

18. Barros AJ, Hirakata VN (2003) Alternatives for logistic regression in cross-sectional studies: an empirical comparison of models that directly estimate the prevalence ratio. BMC Med Res Methodol $3: 21$

19. Spiegelman D, Hertzmark E (2005) Easy SAS calculations for risk or prevalence ratios and differences. Am J Epidemiol 162:199200

20. Julkunen H, Heinonen OP, Knekt P, Maatela J (1975) The epidemiology of hyperostosis of the spine together with its symptoms and related mortality in a general population. Scand $\mathrm{J}$ Rheumatol 4:23-27

21. Kiss C, O’Neill TW, Mituszova M, Szilagyi M, Donath J, Poor G (2002) Prevalence of diffuse idiopathic skeletal hyperostosis in Budapest, Hungary. Rheumatol (Oxf) 41:1335-1336

22. Resnick D, Dwosh IL, Goergen TG et al (1976) Clinical and radiographic abnormalities in ankylosing spondylitis: a comparison of men and women. Radiology 119:293-297

23. Resnick D, Shapiro RF, Wiesner KB, Niwayama G, Utsinger PD, Shaul SR (1978) Diffuse idiopathic skeletal hyperostosis (DISH) [ankylosing hyperostosis of Forestier and Rotes-Querol]. Semin Arthritis Rheum 7:153-187

24. Westerveld LA, van Ufford HM, Verlaan JJ, Oner FC (2008) The prevalence of diffuse idiopathic skeletal hyperostosis in an outpatient population in The Netherlands. J Rheumatol 35:16351638

25. Mata S, Hill RO, Joseph L et al (1993) Chest radiographs as a screening test for diffuse idiopathic skeletal hyperostosis. J Rheumatol 20:1905-1910

26. Jun JB, Joo KB, Her MY et al (2006) Femoral bone mineral density is associated with vertebral fractures in patients with ankylosing spondylitis: a cross-sectional study. J Rheumatol 33:1637-1641

27. Vosse D, Landewé R, van der Heijde D, van der Linden S, van Staa TP, Geusens P (2009) Ankylosing spondylitis and the risk of fracture: results from a large primary care-based nested case control study. Ann Rheum Dis 68(12):1839-1842

28. Eser P, Bonel H, Seitz M, Villiger PM, Aeberli D (2010) Patients with diffuse idiopathic skeletal hyperostosis do not have increased peripheral bone mineral density and geometry. Rheumatol (Oxf) 49:977-981

29. Westerveld LA, Verlaan JJ, Oner FC (2009) Spinal fractures in patients with ankylosing spinal disorders: a systematic review of the literature on treatment, neurological status and complications. Eur Spine J 18:145-156

30. Kiss C, Szilagyi M, Paksy A, Poor G (2002) Risk factors for diffuse idiopathic skeletal hyperostosis: a case-control study. Rheumatol (Oxf) 41:27-30

31. Mader R, Lavi I (2009) Diabetes mellitus and hypertension as risk factors for early diffuse idiopathic skeletal hyperostosis (DISH). Osteoarthritis Cartilage 17:825-828

32. Sarzi-Puttini P, Atzeni F (2004) New developments in our understanding of DISH (diffuse idiopathic skeletal hyperostosis). Curr Opin Rheumatol 16:287-292

33. Sencan D, Elden H, Nacitarhan V, Sencan M, Kaptanoglu E (2005) The prevalence of diffuse idiopathic skeletal hyperostosis in patients with diabetes mellitus. Rheumatol Int 25:518-521 